\title{
Magnetic reconnection in flux-tubes undergoing spinning footpoint motions
}

\author{
A. L. Wilmot-Smith and I. De Moortel \\ School of Mathematics and Statistics, University of St Andrews, North Haugh, St Andrews, Fife, KY16 9SS, UK \\ e-mail: antonia@mcs.st-and.ac.uk \\ Received 12 March 2007 / Accepted 12 May 2007

\section{ABSTRACT}

\begin{abstract}
Aims. Photospheric motions acting on the coronal magnetic field have the potential to build up huge amounts of magnetic energy. The energy may be released through magnetic reconnection, and so a detailed understanding of the 3D process is crucial if its implications for coronal heating are to be fully addressed.

Methods. A 3D MHD experiment is described in which misaligned magnetic flux tubes are subjected to simple spinning boundary motions.

Results. The resulting shear between adjacent flux systems generates a twisted central separator current sheet that extends vertically throughout the domain. Current density is amplified to a sufficient extent that reconnection begins, and occurs everywhere along the separator current sheet, while the separatrix current sheets that exist in the early stages of the experiment are found to be unimportant in the systems dynamical evolution. In $2 \mathrm{D}$ cross-sections, the reconnection process exhibits many similarities to the regime of flux pile-up reconnection.
\end{abstract}

Key words. magnetohydrodynamics (MHD) - Sun: magnetic fields - Sun: corona - Sun: activity

\section{Introduction}

Magnetic reconnection plays a fundamental role in the evolution of astrophysical, solar and terrestrial plasmas. It allows for a change in the topology of the magnetic field and, as such, is the only process that allows topologically bound magnetic energy to be released. Reconnection is thought to play an important role in coronal heating, although modelling the process continues to present a great challenge to theorists. Photospheric motions act to displace magnetic flux tubes anchored there, providing a Poynting flux through the base of the solar corona. However, the high magnetic Reynolds numbers of the corona require very steep gradients in the magnetic field to be built up before non-ideal processes can become important, so allowing for magnetic reconnection and associated energy release. Accordingly, models rely on the large-scale motions producing small-scale structure (Sweet 1958; Parker 1957). This may occur through the action of simple photospheric flows on complex coronal fields (Longcope 1996; Priest et al. 2002), by complex photospheric flows acting on simple coronal fields (Parker 1972; van Ballegooijen 1986; Galsgaard \& Nordlund 1996), or by a combination of these (and possibly other) processes.

There is a growing volume of observational evidence that suggests reconnection is a ubiquitous process in the corona. For example, Tsuneta (1996) reported on an example of large-scale reconnection, with a Yohkoh SXT observation of a developing system of magnetic loops connecting active regions on either side of the equator. Madjarska et al. (2004) observed bidirectional jets at the boundaries of coronal holes and inferred the presence of magnetic reconnection along these boundaries. An analysis of the magnetic field in the quiet-Sun, carried out by Close et al. (2004), suggested the timescale for recycling of the quiet-Sun coronal field could be as short as $1.4 \mathrm{~h}$.
These reconnection events are inherently three-dimensional (3D), but while much of reconnection theory is based on twodimensional (2D) models, several important properties of 2D reconnection no longer apply in 3D. For example, in 2D, reconnection can only occur at an X-type null-point of the magnetic field while in $3 \mathrm{D}$ there are no such topological restrictions; indeed reconnection may occur even when no magnetic null-points are present within the domain (Hornig \& Priest 2003). Separator reconnection (Priest \& Titov 1996) can take place across magnetic separators (field-lines connecting two null-points). Spine and fan reconnection are both associated with isolated null-points, with their occurrence dependent on the orientation of the current at the null (Pontin et al. 2004, 2005). The location of reconnection in 3D will depend on the location of any non-ideal terms, such as current concentrations, that can break the frozen-in field condition. Magnetic separators are field-lines connecting two null-points that, in general, lie at the boundary of four regions of differing flux connectivity. They are thought, therefore, to be favourable locations for current sheet build-up (Longcope \& Cowley 1996) and so highly likely sites for reconnection.

In addressing the question of maintenance of the $1 \mathrm{MK}$ quiet-Sun coronal temperature, several numerical simulations have focused on elementary heating events, considering the effect of simple footpoint motions on current sheet formation and reconnection. The relative motion of two magnetic sources which are initially unconnected but have an overlying background magnetic field has been extensively numerically modelled (Galsgaard et al. 2000; Parnell \& Galsgaard 2004; Galsgaard \& Parnell 2005; Haynes et al. 2007) and the flux sources found interact through separator reconnection.

We present here 3D numerical simulations of an elementary heating event, which builds on the papers of De Moortel \& Galsgaard $(2006 a, b)$. In that series of papers the authors consider 


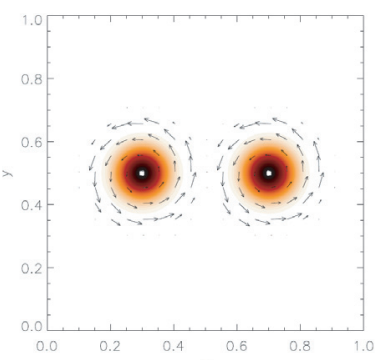

(a)

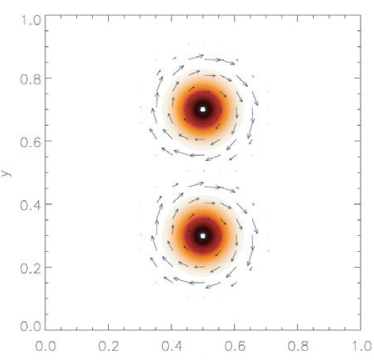

(b)

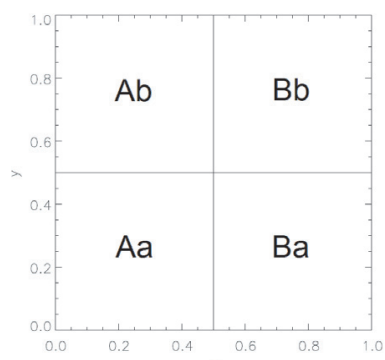

(c)
Fig. 1. Contour plot illustrating $|\boldsymbol{B}|$ on a) the lower and $\mathbf{b}$ ) the upper boundaries of the domain. Superimposed are vectors of the imposed spinning driving velocity in the same planes. The flux sources are labelled $A$ (leftmost source) and $B$ (right-most source) on the lower boundary and $a$ (lower source) and $b$ (upper source) on the upper boundary. As shown for the $z=0.5$ plane in $\mathbf{c}$ ), there are initially four regions of differing magnetic flux connectivity in the domain, with flux connecting sources $A$ and $a$ labelled $A a$, and similarly for $A b, B a$ and $B b$. the interaction of two magnetic flux tubes as they are subjected to two distinct types of motions imposed on the boundary footpoints. The first is a large-scale rotating motion in which both of the footpoints on each of the boundaries are rotated and the second a small-scale spinning motion in which each footpoint is spun while its position remains fixed. The magnetic flux tubes in the spinning case (De Moortel \& Galsgaard 2006b) are initially perfectly aligned and hence remain so throughout the experiment. The experimental setup taken is therefore non-generic, representing a situation extremely unlikely to arise in the solar context. In the initial potential field extrapolation of perfectly aligned flux sources there are two flux-domains with a single boundary between them, while in any other situation where the flux tubes are not perfectly aligned there are four flux-domains and correspondingly four boundaries between domains. It is not clear whether in the misaligned case, with its additional boundaries and likely sites for current-sheet formation, the nature of the reconnection taking place will be the same as that found in the perfectly aligned case. In particular, the rate of reconnection could be quite different, with corresponding implications for coronal heating.

In this paper we examine the relevant case for the solar corona where the magnetic flux tubes are misaligned, imposing the same spinning motions on the tube footpoints as De Moortel \& Galsgaard (2006b). One aim of the paper is to examine the nature of the $3 \mathrm{D}$ reconnection process that takes place (Sect. 3). This was not considered in detail by De Moortel \& Galsgaard (2006b) since they chose instead to take a number of different experimental setups and examine similarities and differences between them in terms of current evolution, flux connectivities and energetics. In Sect. 4 we then use some comparisons between data of one of the cases of De Moortel \& Galsgaard (2006b) and our results to see how the nature of the reconnection differs for the two examples and we conclude in Sect. 5. We begin in the next section by briefly describing the experimental setup.

\section{Model setup}

The dimensions of the domain are $(1,1,1)$. We place two negative sources (which we label $A$ and $B$ for convenience) on its lower boundary, aligned with the $x$-axis, and two positive sources (labelled $a$ and $b$ ) on its upper boundary, aligned with the $y$-axis:

$B_{z}(x, y, z=0)=\mathrm{e}^{-r_{1}^{2} / r_{0}^{2}}+\mathrm{e}^{-r_{2}^{2} / r_{0}^{2}}$,

$B_{z}(x, y, z=1)=\mathrm{e}^{-r_{3}^{2} / r_{0}^{2}}+\mathrm{e}^{-r_{4}^{2} / r_{0}^{2}}$,

where $r_{0}=0.065, r_{1}^{2}=(x-0.3)^{2}+(y-0.5)^{2}, r_{2}^{2}=(x-0.7)^{2}+$ $(y-0.5)^{2}, r_{3}^{2}=(x-0.5)^{2}+(y-0.3)^{2}$, and $r_{4}^{2}=(x-0.5)^{2}+$ $(y-0.7)^{2}$. These sources are shown in Fig. 1. Using these boundary conditions, a potential magnetic field is calculated to fill the domain and imposed as an initial condition. The four distinct flux domains that result from the misalignment of the four sources are shown for the central plane $(z=0.5)$ in Fig. 1c.

Throughout the experiment each of the four flux sources is spun, those on the lower boundary in a counter-clockwise direction and those on the upper boundary in a clockwise direction:

$$
\begin{aligned}
v_{\theta}(r, z=0)= & v_{0} r_{1}\left[1+\tanh \left(p\left(1-q r_{1}\right)\right)\right] \\
& +v_{0} r_{2}\left[1+\tanh \left(p\left(1-q r_{2}\right)\right)\right] \\
v_{\theta}(r, z=1)= & -v_{0} r_{3}\left[1+\tanh \left(p\left(1-q r_{3}\right)\right)\right] \\
& -v_{0} r_{4}\left[1+\tanh \left(p\left(1-q r_{4}\right)\right)\right]
\end{aligned}
$$

where $v_{0}=0.02222, p=16.8$ and $q=5.6$. This velocity has been chosen such that the shape of the flux sources on the boundaries is largely maintained as they are spun. In the descriptions of the experimental results we refer to the spin angle, $\theta$; this measures the angle in radians by which the sources have been spun from their initial positions. The simulation is carried out for $\theta \in[0,2.64]$. Since the spinning motion is imposed in opposite directions on both boundaries the flux tubes themselves are subjected to an effective spinning angle of $2 \theta$. Note also that the chosen driving velocity is very slow compared with the typical Alfvén velocity.

The dynamical evolution of the system is obtained by using a numerical code (as described in detail by Nordlund \& Galsgaard 1997) to solve the non-ideal MHD equations in the same manner as that of De Moortel \& Galsgaard (2006a,b). Periodic boundary conditions are imposed on the sides of the box with driven upper and lower boundaries and the grid resolution is $128^{3}$. The resultant evolution of the system is described in the following sections.

\section{Experimental results}

In this section we focus on the basic dynamical evolution of the system, placing particular emphasis on the reconnection mechanism that takes place. We consider first, in Sect. 3.1, the character of the magnetic flux connectivity and how it develops with spin angle. We proceed in Sect. 3.2 to describe the nature and evolution of the current concentrations before considering the plasma velocities and implications for the reconnection mechanism in Sect. 3.3.

\subsection{Magnetic flux connectivities}

The effect of the spinning footpoint motions is to drive the magnetic field away from the initial potential state and results in reconnection between the magnetic flux tubes. Figure 2 gives 


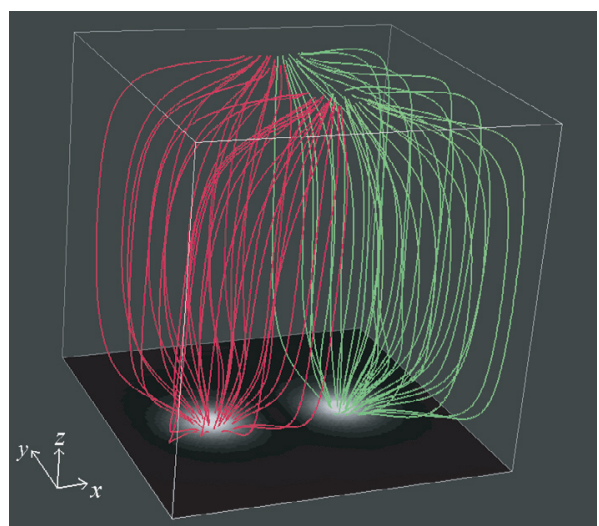

(a)

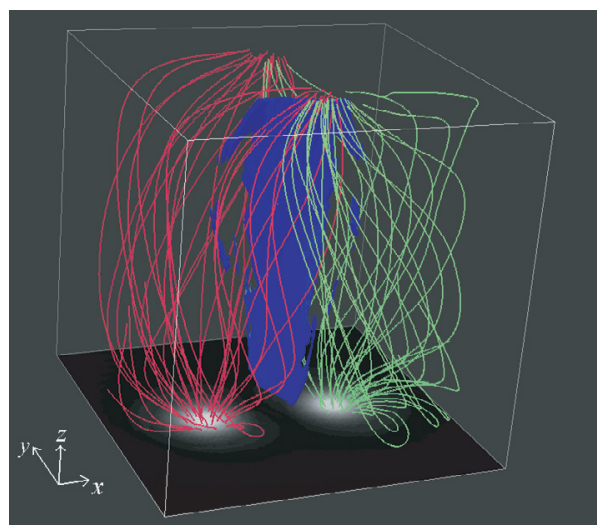

(c)

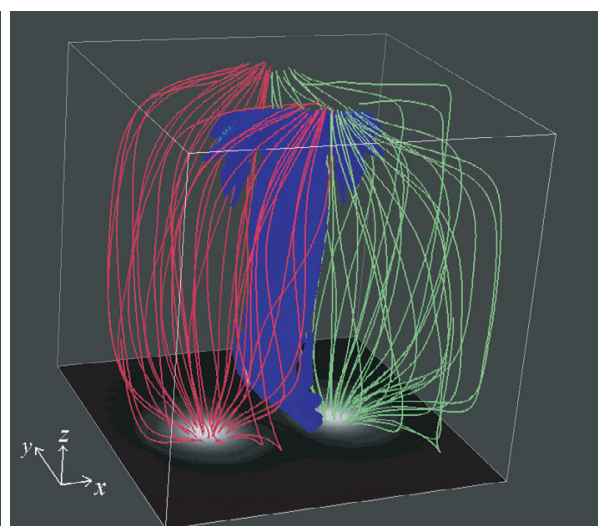

(b)

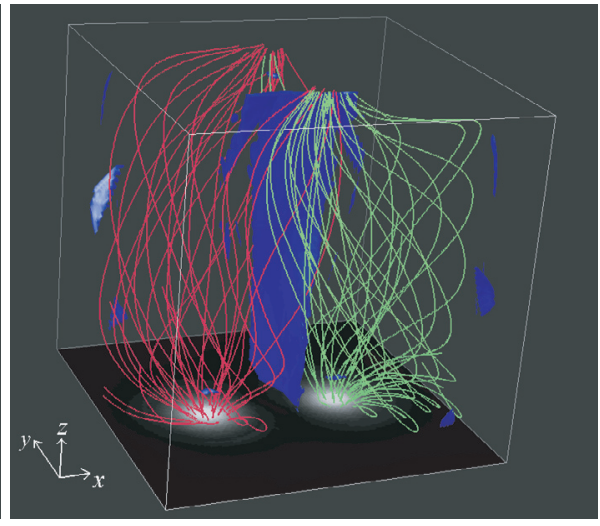

(d)

Fig. 2. Selected field lines traced from the two sources on the lower boundary, at spin angles a) $\theta=0$ b) $\theta=0.79$ c) $\theta=1.87$ and d) $\theta=2.64$. Those traced from source $A$ are coloured red and those from source $B$ coloured green. The field lines are seen to become increasingly twisted with spin angle and, in addition, it is seen that the initially equal distribution of flux from a single source on the lower boundary between both sources on the upper boundary becomes unequal with increasing spin-angle. Over-plotted are isosurfaces of current; a twisted current sheet is seen to form in the centre of the domain.

a qualitative overview of how the magnetic flux in the domain evolves as the sources on the boundary are spun. Some illustrative field lines have been traced from the two sources on the lower boundary and coloured red if they are associated with source $A$ and green for source $B$. In the initial potential field, shown in Fig. 2a, flux from each of the lower sources is divided equally between the two upper sources (and vice versa). As the sources are spun the magnetic flux in the domain becomes increasingly twisted, as seen in the sequence of images Fig. 2(b-d). In addition, the magnetic flux connectivities of the sources change with increasing spin angle; by the end of the experiment, flux from source $A(B)$ is predominately connected to source $b(a)$. Superimposed on the same diagrams are (the same) isosurfaces of strong current. Note that, for clarity, current in the three grid cells closest to each of the boundaries has been removed from the diagrams. Early in the experiment, a twisted current sheet forms in the centre of the domain, extending vertically throughout the box, and this current sheet persists throughout the simulation (see Sect. 3.2 for more details).

Considering the evolution of connectivity of the magnetic sources themselves, we note first that the flux sources are not ideal and so a small amount of slippage occurs within them. This is not important for the dynamical evolution of the system but means that we are unable to follow the evolution in time of individual field lines exactly at these locations. Instead, for each spin-angle we trace a large number of field lines from the sources on the lower boundary. For each such field line we deduce its magnetic connection on the upper boundary and the amount of magnetic flux associated with it. In Fig. 3, field lines have been traced from source $A$ to the upper boundary and coloured dark blue if they are connected to source $b$ (i.e. if their magnetic connectivity is of type $A b$ ), light blue if they are connected to source $a$ (i.e. if their magnetic connectivity is of type $A a$ ) and red if they leave the box (which is allowed because of the periodic nature of the side boundaries). The total magnetic flux of type $A a$ is seen to decline with spin angle, whilst that of type $A b$ is seen to increase.

This information can be used to deduce the percentage of flux from a source on the lower boundary connected to each of the two sources on the upper boundary. Figure 4 shows the change in these quantities with spin angle, together with the percentage of flux which leaves the box. Reconnection begins at spin angle $\theta=0.40$ and the amount of flux with connectivity $A a(A b)$ subsequently decreases (increases) linearly with spin angle until $\theta \approx 2.3$, close to the end of the experiment, when flux begins to leave the box. At the end of the experiment $22.3 \%$ of the flux from source $A$ is connected to source $a$, so we can therefore deduce that at least $27.7 \%$ of the flux in the source has been reconnected by spin angle $\theta=2.64$. These quantities set a lower bound on the amount of reconnected flux which will be greater if flux of type $A b$ reconnects with that of type $B a$ at any stage in the experiment (to form additional flux of type $A a$ ).

Given that the magnetic flux connectivity inside the domain is changing as the spin-angle increases, we examine how the changes become evident in the central plane, $z=0.5$. We trace a large number of the field lines passing through the entire central plane, determine the connection of each field line on both the upper and the lower boundary, and assign the field line a colour according to its magnetic connectivity. Note that the tracing is now begun at the central plane rather than the source footpoints. By showing these colours in the central plane we generate a diagram in which the central plane is colour-coded according to the 

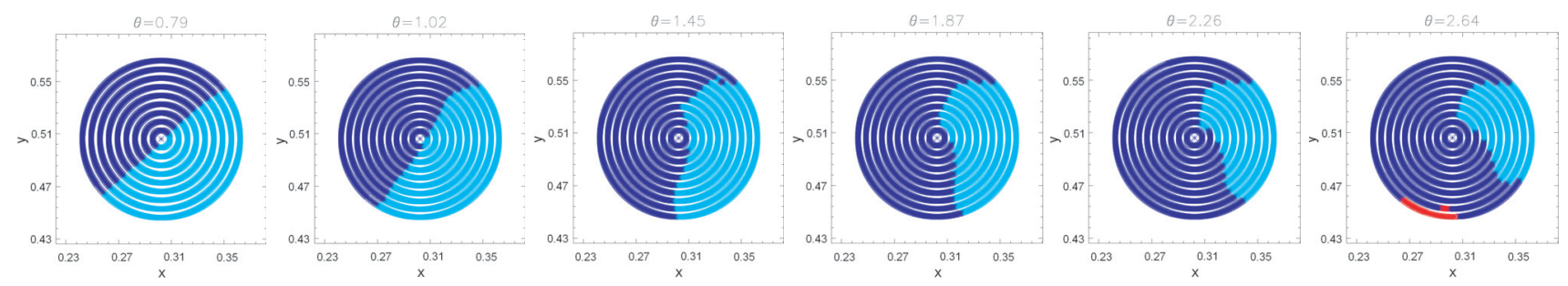

Fig. 3. Magnetic flux connectivity of source $A$ with spin angle. Flux with connectivity $A a$ is shown in light blue, flux with connectivity $A b$ is shown in dark blue and flux which leaves the box in red. Due to the symmetry of the system the flux connectivity of source $B$ follows the same pattern, with connectivity type $B a$ becoming dominant.

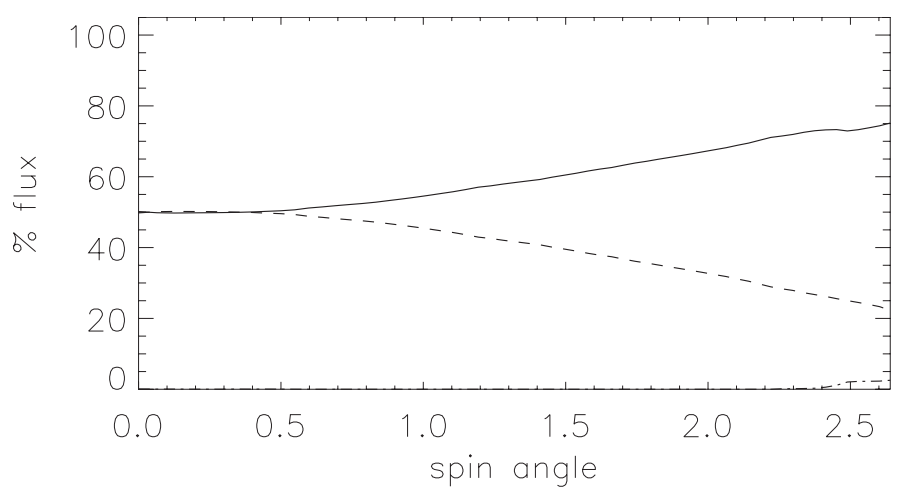

Fig. 4. Change in magnetic flux connectivity with spin angle of source $A$ $(B)$ on the lower boundary. The solid line shows the percentage of flux with connection $A b(B a)$, the dashed line the percentage of flux with connection $A a(B b)$ and the dot-dashed line the percentage of flux from source $A(B)$ that leaves the box.

magnetic connectivity of the flux that pierces it. Figure 5 illustrates this connectivity for a sequence of increasing spin angles, with contours of electric current superimposed onto the same diagrams to enable us, at a later stage, to determine the role of the current in the flux evolution.

As shown in Fig. 5, even by spin-angle $\theta=0.65$ when just $1.47 \%$ of the magnetic flux of source $A$ has reconnected, the arrangement of flux in the central plane has been significantly altered by the spinning motions. The four types of flux no longer meet at a point, but rather types $B b$ (red) and $A a$ (light blue) meet along a central line with types $A b$ (dark blue) and $B a$ (yellow) no longer coming into contact. Currents have built-up along the boundaries between the various flux domains but remain confined close to the centre of the numerical box in the horizontal direction. As the spin-angle increases, the area of the central plane pierced by flux types $A b$ and $B a$ increases also and at later spin angles $(\theta \geq 1.70)$ the flux types $A a$ and $B b$ are no longer in contact with the boundary, being instead entirely enclosed by the remaining flux types. The strong central current sheet coincides exactly with the boundary between the flux types $A a$ and $B b$ but the weaker "wings" of current emanating from the central twisted sheet do not perfectly outline the remaining boundaries between the various flux types.

Some of the magnetic flux near the boundary of the $z=$ 0.5 plane is not associated with any of the four flux types since we have chosen the particular radius $\left(2 r_{0}\right)$ to define each magnetic source. Since the flux sources are exponentially decaying this radius is artificial and so flux falling outside the radius is not considered to be associated with a particular flux source. In addition, toward the end of the simulation some magnetic flux leaves the box through the side boundaries. Neither of these flux connectivity types have been colour coded.

\subsection{Current evolution}

The spinning motions imposed on the footpoints of the flux sources act to spin up the flux in each of the domains and so generate a shear at each of the interfaces between domains. Comparison with the current contours superimposed on the flux connectivity plots of Fig. 5 confirms that the current sheet shown in Fig. 2 is a separator current sheet (Longcope \& Cowley 1996; Longcope 2001), twisted in the vertical direction as a result of the $\pi / 2$ misalignment of the upper and lower sources. As shown in Fig. 5, four "wings" of current emanate from the central separator current sheet and these wings approximately outline the boundaries between domains.

To examine the nature of the currents within the domain in more detail we consider the central plane, $z=0.5$, and show in Fig. 6 contours of current in that plane for a sequence of increasing spin-angles. The drop in the spinning velocity at the outside of the flux sources results in four "rings" of current at early spin-angles, with the strongest current seen at the intersections of the rings where the interaction of spinning motions produces a shearing effect. By $\theta=0.55$, the separator current sheet together with four wings of current has formed and the initial rings of current have been forced outwards by magnetic pressure. This leads to a build up of current on the side boundaries of the box which ultimately allows flux to leave the box. The wings of current are identified as separatrix current sheets (Priest et al. 2005) early on in the experiment when they perfectly outline the boundaries between the flux domains and lengthen (in the horizontal direction) with spin-angle, extending almost to the boundaries of the domain. The current structure at these early stages of the experiment is therefore similar to that predicted by Green (1965). As reconnection begins and the evolution is no longer quasi-static, the wings of current no longer align with the change of flux connectivity.

By considering the variation of certain quantities along a perpendicular section to the structures, as shown for spin-angle $\theta=1.45$ in Fig. 7, we identify them as contact discontinuities (see, for example, Priest 1982). In order to determine why there is a discrepancy between the locations of the separatrices and of these wings of current we must first consider the nature of both the central separator current sheet and the reconnection mechanism.

Consider now the evolution of the separator current sheet. We will refer to its extent in the $z$-direction as "height", its length in the $x-y$ plane (i.e. along the $y=-x$ line) as "length" and the remaining dimension, its thickness in the $x-y$ plane as "width". As seen in the cross-sections through the current structure (Fig. 6), after its initial formation, the length of the sheet increases with spin angle. The growth in length is almost linear until, at $\theta \approx 1.1$, the ends of the sheet (in the horizontal direction, along the $y=-x$ line) bifurcate to form two Y-type structures 
$\theta=0.00$

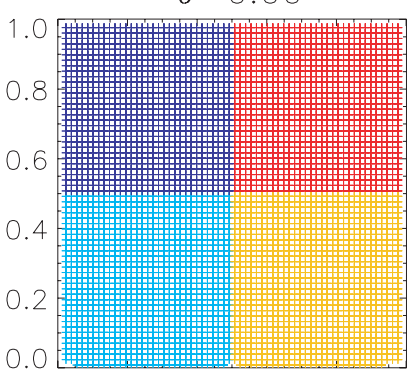

$\theta=1.24$

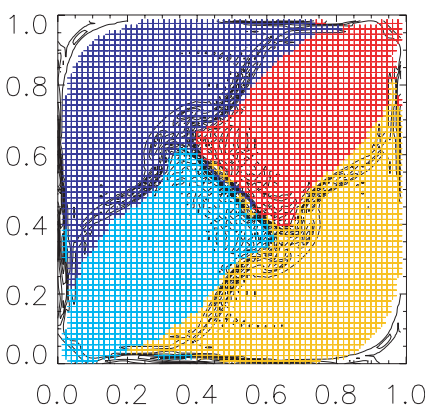

$\theta=0.65$

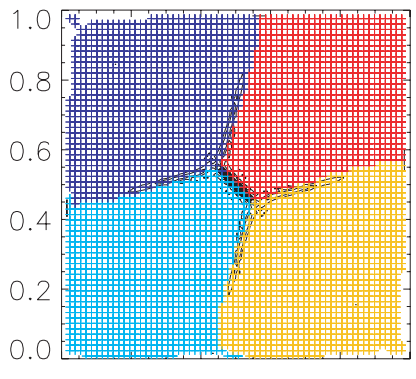

$\theta=2.06$

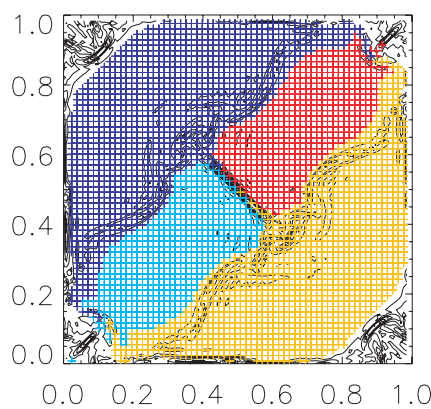

$\theta=0.92$

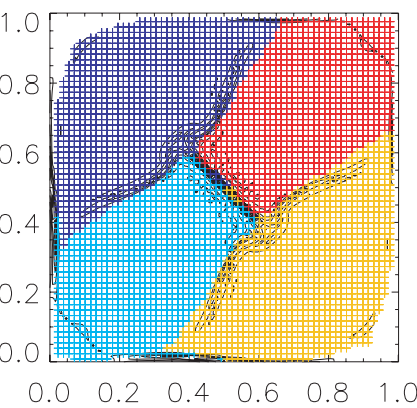

$\theta=2.53$

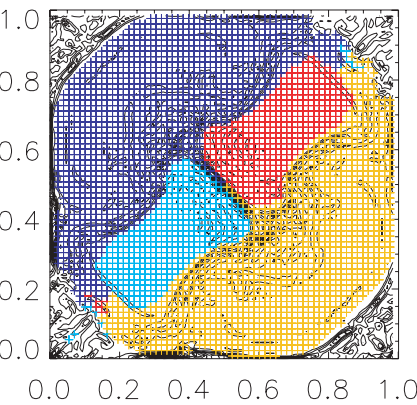

Fig. 5. The figure shows a sequence of images at increasing spin-angles in which the magnetic flux passing through the central plane, $z=0.5$, is coloured according to its magnetic connectivity. Flux with connectivity $A a$ is indicated in light blue, $A b$ in dark blue, $B b$ in red and $B a$ in yellow. Flux not associated with any of these connectivity types is not coloured. Over-plotted are contours of current density in the same plane.

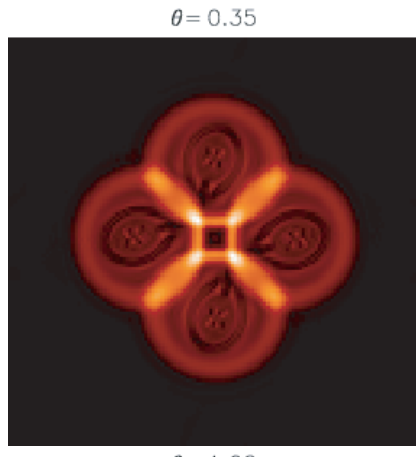

$\theta=1.02$

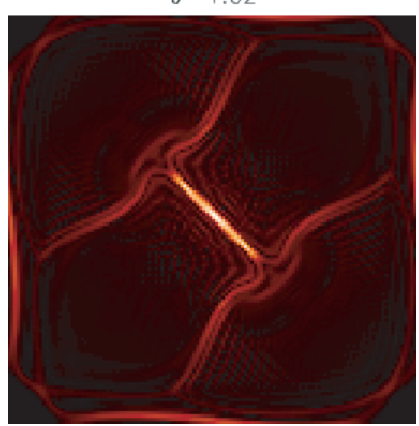

$\theta=0.40$

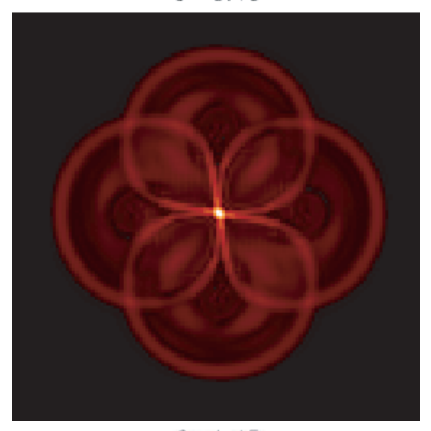

$\theta=1.45$

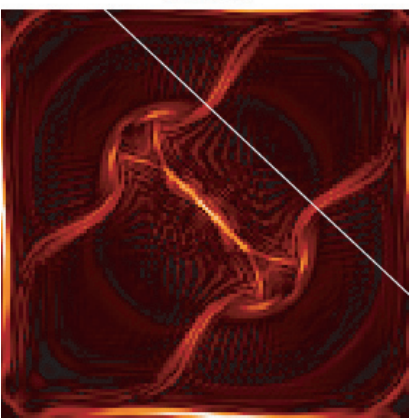

$\theta=0.55$

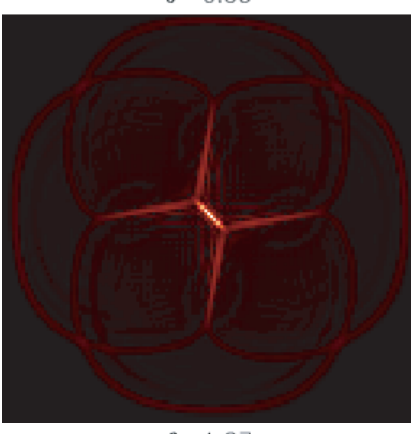

$\theta=1.87$

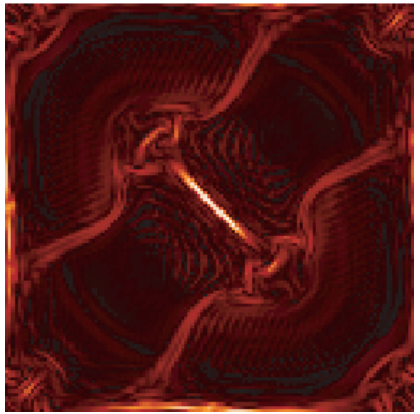

$\theta=0.79$

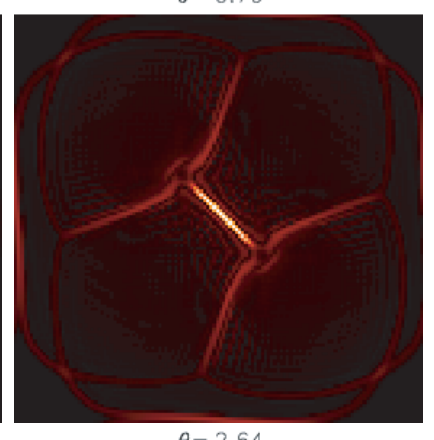

$\theta=2.64$

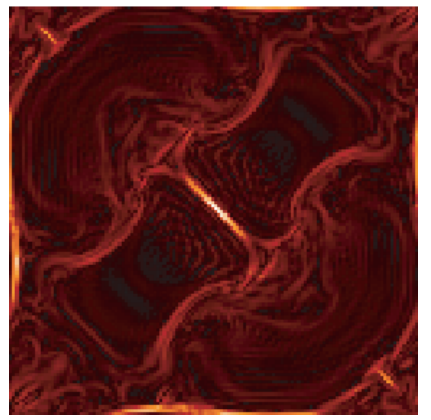

Fig. 6. Sequence of images showing contours of current in the central plane $z=0.5$ at increasing spin angles. Four wings of current are seen to extend from a strong current sheet in the centre of the domain. At later spin-angles Y-shaped cusps are seen to develop at the ends of the current sheet, seen here for example at $\theta=1.45$. (The white line outlines a cross-section described later in the text.)

which lie along the separatrices of the field. No further lengthening of the sheet occurs, indeed it shrinks slowly with further increase in spin angle.

The current within the sheet is predominately in the $\hat{z}$-direction and this component changes sign at both ends of the sheet, as shown in Fig. 8 (where spin-angle $\theta=2.26$ has been considered as an example), indicating the presence of a reverse current. In two-dimensional situations, reversed currents near the ends of diffusion regions have been observed in numerical experiments (Biskamp 1986) and are seen to slow down the 

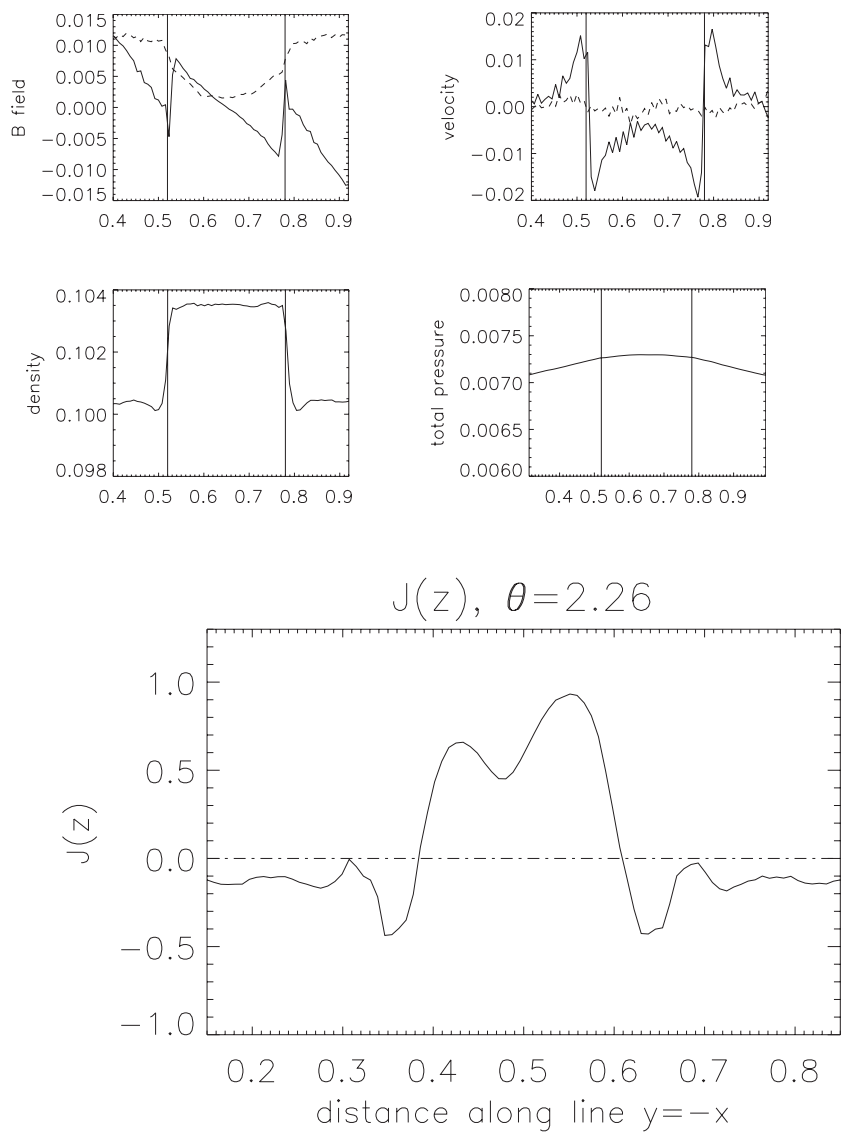

Fig. 8. Strength of the $z$-component of the electric current in the central plane $z=0.5$ along the line $y=-x$ which passes along the central separator current sheet. A spike of reversed current is seen at both ends of the sheet.

outflowing jets in these cases. We therefore proceed to examine the nature of the plasma velocities to determine whether the same occurs in this 3D situation.

\subsection{Plasma velocities and reconnective behaviour}

The $v_{z}$ velocity component component is an order of magnitude less than both the $v_{x}$ and $v_{y}$ components and therefore we consider here the plasma flow in the $x-y$ plane. Figure 9 shows vector field plots of $\left(v_{x}, v_{y}\right)$ in the central plane at various spinangles. At early spin-angles, $\theta=0.35$ for example, the velocity in the central plane reflects the driving velocity imposed on the upper and lower boundaries, with four counter-rotational flow regions present. The intersection of these regions results in a stagnation-flow that is stronger than the remaining rotational components. It is this stagnation-flow that dominates the later velocity profiles. Plasma flows into, and is ejected from, the separator current sheet, with the inflow streamlines being curved and diverging and the outflow, particularly at later spin-angles, diverted out along the separatrices of the field.

Considering the nature of the inflow region in more detail and paying particular attention to the magnetic field and gas pressure we note that the gas pressure decreases as the plasma flows in toward the separator current sheet, suggesting the inflow is undergoing an expansion. In addition the reverse behaviour is observed in the magnetic pressure profile which increases toward the current sheet so that the expansion may be further characterised as of the slow-mode type. $>$ From this combination

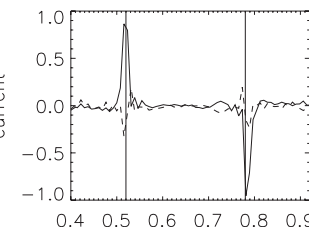

Fig. 7. Tangential (solid lines) and normal (dashed lines) components of the magnetic field, velocity and current (upper plots) together with the density, total pressure and $|\nabla \times v|$ (lower plots) along the line $y=1.3-x$ at spin-angle $\theta=$ 1.45 for the central plane $z=0.5$. The vertical lines denote the location of the current "wings" along that line (as seen in Fig. 6). The presence of a normal field component across the structures, absence of a plasma flow across them together with the continuous pressure variation but jumps in density are evidence for a contact discontinuity at these locations. of characteristics, together with the current profile and plasma flows, we deduce that the situation is strongly reminiscent of the flux pile-up regime (Priest \& Forbes 1986), with its characteristically long diffusion regions. This model was extended further in the non-uniform theory of Priest \& Lee (1990) to also take reversed current spikes and separatrix plasma jets into account. Shocks in their (incompressible) model are rather weak and indeed we cannot consider any of the structures in this $3 \mathrm{D}$ experiment as true shocks. There are, however, several differences between the $2 \mathrm{D}$ theory and this $3 \mathrm{D}$ model. In the reconnection process, magnetic flux of types $A a$ and $B b$ are brought together and reconnect across the central separator current sheet to form flux of types $A b$ and $B a$. However, as distinct from the 2D theory, in this $3 \mathrm{D}$ case the magnetic field has an O-type structure in cross-sections of constant $z$ (Fig. 10b) and it is the vertically orientated flux that is reconnected. Thus reconnection can occur along the entire height of the separator current sheet with the exact location depending on where the flux comes into contact with the sheet. Figure 10a shows four particular field-lines which illustrate the reconnection process, together with contours of current in the central plane. The sheared magnetic flux in the inflow regions (as illustrated by the red field-line of type $B b$ and light blue field-line of type $A a$ ) is carried into the central current sheet where it reconnects. The strong outflow then carries the reconnected flux out of the current sheet and this post-reconnection flux has less shear (as illustrated by the dark blue field-lines of type $A b$ and yellow field-line of type $B a$ which are almost straight). Note that the field-lines shown in this figure are for illustrative purposes only and all taken at the same spin-angle, i.e. they do not represent the same pre- and post-reconnection field-lines

The magnetic flux that pierces the central plane close to the contact discontinuity is twisted in such a way that it passes through the separator current sheet towards the top or bottom of the box and reconnects there. Thus the reason behind the misalignment of the wings of current (identified with contact discontinuities) and separatrices of the field becomes evident. Reconnection is taking place along the entire height of the separator current sheet but the resultant changes of flux connectivity are not immediately apparent at different heights within the domain. The contact discontinuities themselves are an artifact of the initial flux distribution and the spinning motions imposed on that distribution. They outline the divide between flux types which would have existed had no reconnection taken place.

\section{Discussion}

Examining in more detail the flux connectivity diagrams for the mid-plane (shown in Fig. 5), an interesting pattern of 

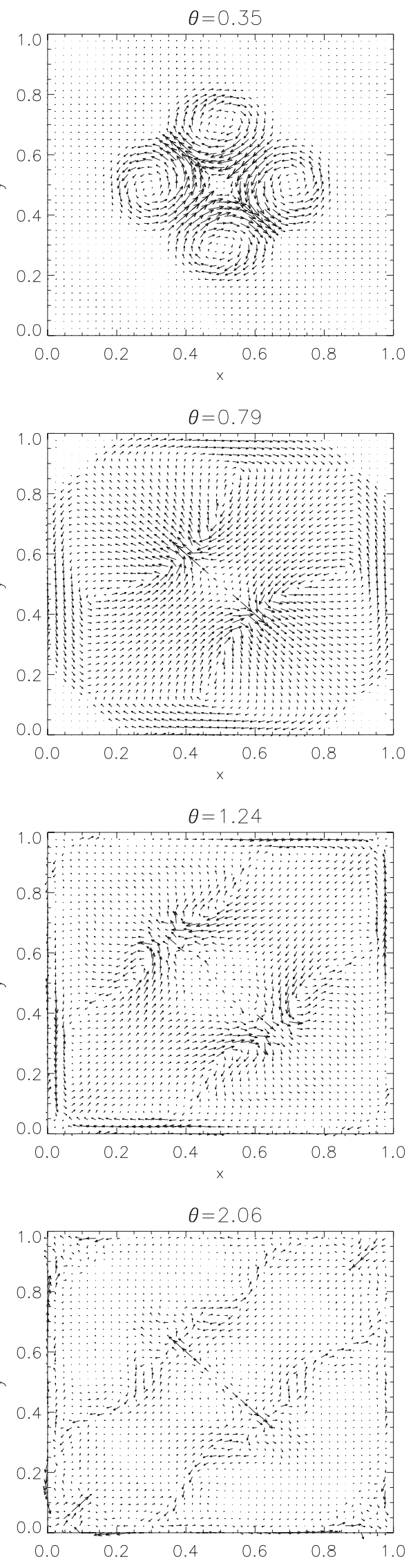

Fig. 9. Plasma flows in the central plane, $z=0.5$ for a sequence of increasing spin-angles showing the key stages in the velocity evolution. A stagnation flow forms with strong outflow jets along the central current sheet.

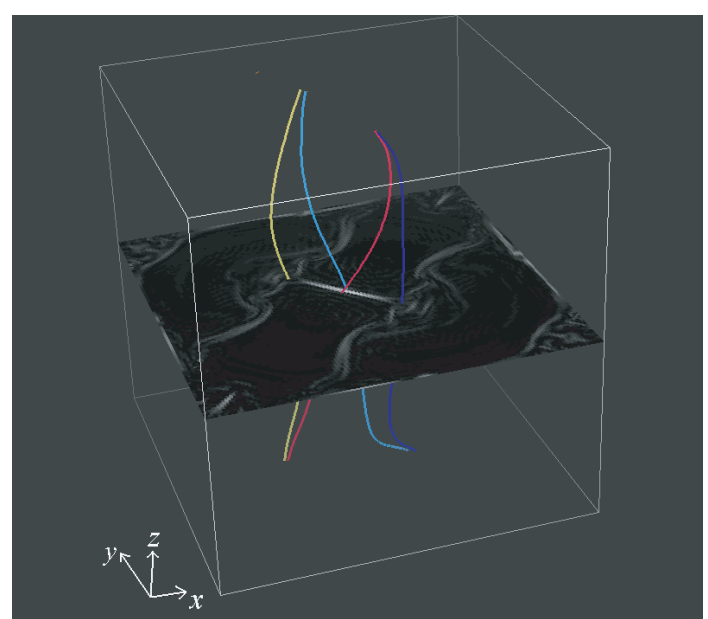

(a)

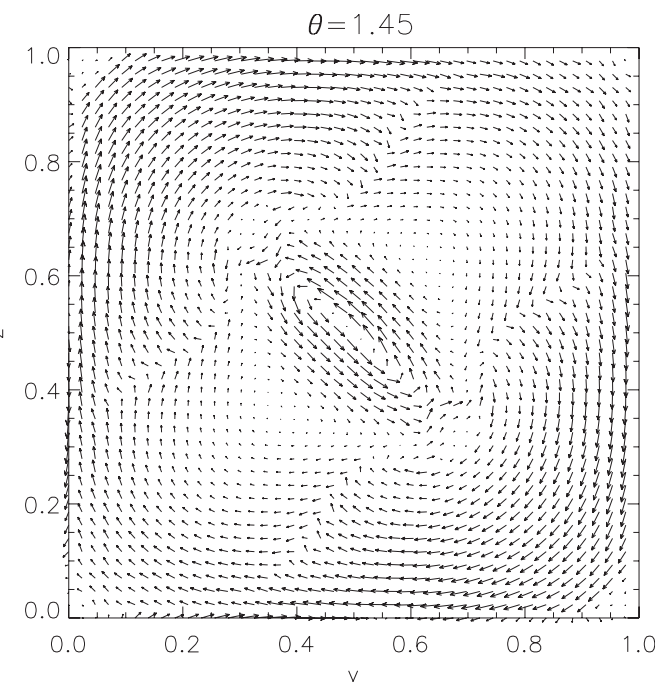

(b)

Fig. 10. a) Four illustrative field-lines being carried into the central current sheet before reconnection (red, light blue) and away from the sheet having reconnected (dark blue, yellow), together with contours of current in the central plane. The post-reconnection field lines are seen to have less shear than the pre-reconnection lines. b) An X-type field structure is present only in vertical cross-sections, while in horizontal sections, such as that illustrated here, the field has an O-type topology.

behaviour is seen within the central current sheet at intermediate spin-angles ( $\operatorname{see} \theta=0.92,1.24$ for example). As an illustration, an enlargement of this region is shown at spin-angle $\theta=1.19$ in Fig. 11 (left). Although in Sect. 3 we have somewhat loosely referred to the "separator current sheet", this diagram, with its regions of 2D isolated flux-connectivity type, indicates the topology of the region is really very complex, with multiple separators perhaps present, and only becomes simple again in later stages of the experiment (as shown in Fig. 11 (right)). This effect does not result from a lack of resolution of the current sheet in the later stages; the current sheet remains well-resolved throughout the experiment through the use of hyper-resistivity (see Nordlund $\&$ Galsgaard 1997). A detailed investigation into the magnetic topology of a particular 3D MHD reconnection experiment was carried out by Haynes et al. (2007), where a sequence of bifurcations was identified which resulted in the initial field topology becoming increasingly complex, before eventually simplifying 

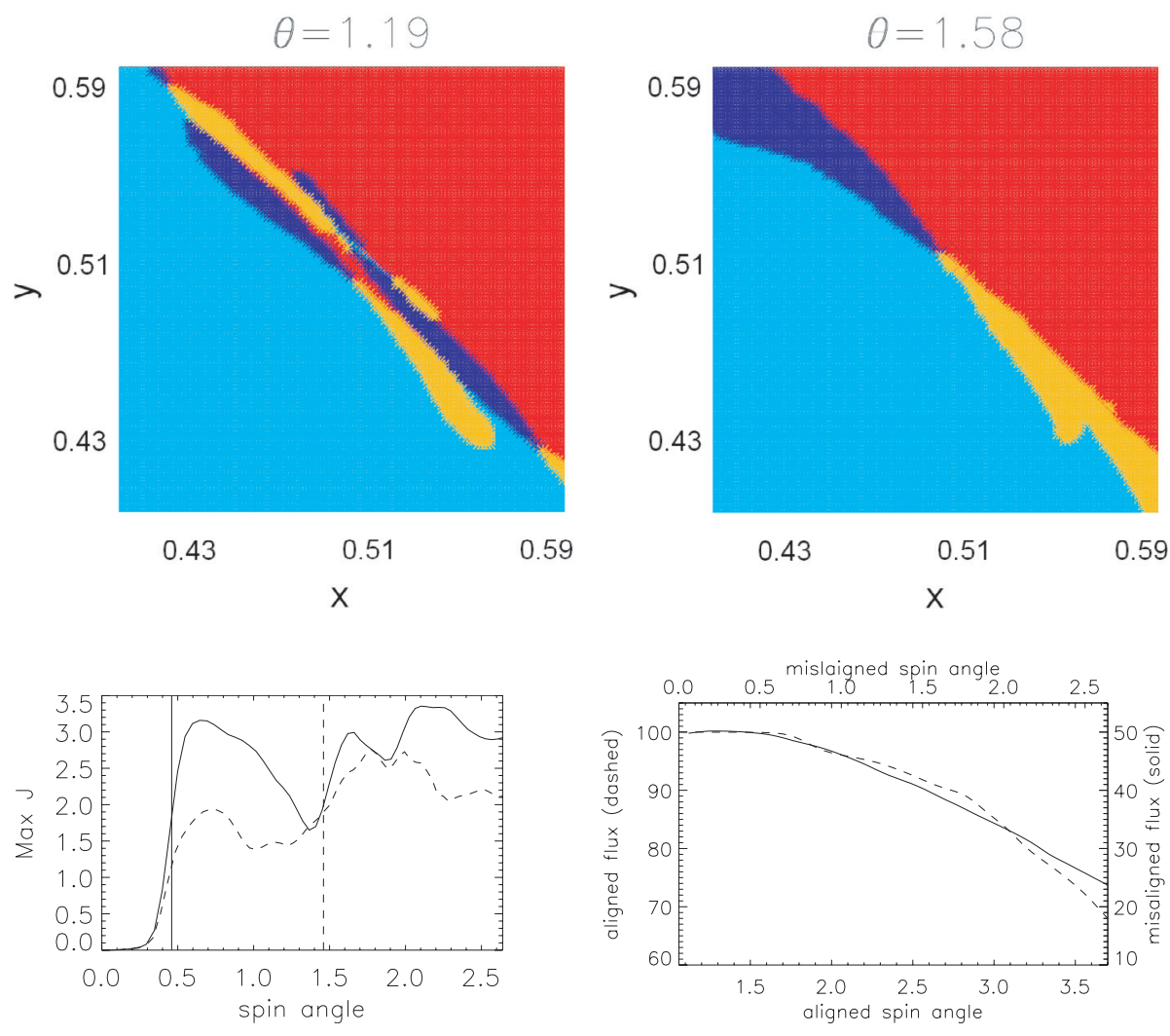

Fig. 11. Flux connectivities in a central square within the mid-plane encompassing the nonideal region, for (left) $\theta=1.19$ and (right) $\theta=1.58$. The colour scheme is the same as that for Fig. 5. A complex topology is present in the early stages of the experiment, becoming much simpler as the spin-angle increases. in the later stages of the experiment. From the preliminary investigations presented here, we expect a similarly complex topology It would therefore be worthwhile to carry out such an investigation and consider the implications for the reconnection process. This, however, is beyond the scope of the simple descriptive content of this paper.

As discussed in Sect. 1 one of the motivations to consider reconnection in these misaligned flux tubes is to make a comparison with the case of the same spinning footpoint motions imposed on perfectly aligned flux tubes, as described by De Moortel \& Galsgaard (2006b) and, in particular, to examine how the nature of reconnection differs between the two situations.

The spin-angle for the onset of reconnection is $\theta=1.46$ in the aligned case and $\theta=0.40$ in this misaligned situation. The difference in spin-angle $(\Delta \theta=1.06)$ corresponds to a time difference in the solar corona of $0.6 \mathrm{~h}$ (for a discussion of how the non-dimensional quantities described in this experiment relate to coronal parameters see De Moortel \& Galsgaard 2006a). This is a significant difference between the two cases given that the coronal recycling time is estimated to be as little as $1.4 \mathrm{~h}$.

The likely reason behind the disparity in reconnection onset times can be found by comparing the plasma velocities and build-up of current in the two experiments. In the misaligned case, the imposed boundary flows propagate into the box in such a way as to form a stagnation-flow early in the experiment, as shown at $\theta=0.35$ in Fig. 9. However, in the aligned case, the counter-spinning boundary flows effectively cancel as they propagate into the mid-plane, and so a stagnation-flow is only initiated at a later stage through the effect of magnetic pressure. Stagnation-flows have the effect of amplifying current concentrations and, accordingly, a build-up of sufficient current to allow for reconnection to take place occurs sooner in the misaligned case. The evolution of maximum $|\boldsymbol{j}|$ in a central square of side-length 0.4 and in the mid-plane $(z=0.5)$ with spin-angle is shown, for both experiments, in Fig. 12(left). Although the initial current development begins in both experiments at the same spin angle, the initial growth is faster for the misaligned case. In both situations there then follows a period where the maximum current decreases with spin angle before undergoing a second phase of increase. In the aligned case it is only in this second phase of current growth that reconnection begins (vertical dashed line).

The next important comparison is in how, once initiated, the rate of reconnection differs between the two setups. In order to make this comparison we consider how the percentage of flux with certain connectivities changes with spin angle. In the misaligned case flux with connectivity $A a$ is considered. This fluxtype initially constitutes $50 \%$ of the flux from source $A$, and the percentage decreases with spin-angle after reconnection begins. For the aligned case the percentage of flux remaining at its original flux source is considered (for one of the sources on the lower boundary). This is initially $100 \%$ of the flux in the source and again decreases with spin-angle after the onset of reconnection. These quantities are represented in Fig. 12(right) where the $x$-axes for both cases have been aligned in such a way that the onset of reconnection is coincident. We see that during the initial phase of reconnection the rate of decrease of flux of the considered connectivity is very similar in both cases. This suggests that the same reconnection mechanism may be responsible for the evolution of both systems. As spin-angle increases a change in the gradient of flux connectivity occurs in the aligned case, at aligned spin-angle $\theta=2.8$, indicating flux is now changing connectivity faster. Examining the flux evolution in that experiment in more detail we observe that this discrepancy is due to flux leaving the box, i.e. additional reconnection occurring across the boundaries of the domain, rather than a change in the reconnection mechanism within the central current sheet. Thus it is interesting to note that although the current sheet has a greater cross-sectional length in the aligned than the misaligned case (with the additional flux domains in the misaligned case 
restricting current sheet growth), this does not result in a different rate of reconnection.

One notable difference found between the two experiments is in the geometry of the central current sheet. In the aligned case the sheet is straight, while in the misaligned case a twisted sheet forms as a result of the $\pi / 2$ difference in orientation of the upper sources. In the misaligned case the initial potential field contains four distinct flux domains which allows for the subsequent development of separatrix current sheets; these are necessarily absent in the aligned experiment (or, alternatively, can be considered as coincident with the separator current sheet). However we have shown that the separatrix current sheets in the misaligned case, which later become contact discontinuities, are not important in the reconnection process itself. The comparable reconnection rates found in the two experiments confirms this to be the case and is further evidence that the reconnection process is concentrated in the separator current sheet.

Finally we note that there are several limitations in the experimental setup. Perhaps the most important of these is in the plasma $\beta$ which, since the field strength decreases rapidly moving away from the upper and lower boundaries toward the centre of the domain whilst the gas pressure profile is initially uniform, is significantly higher than that found in the solar corona. In addition, the experiment ends at $\theta=2.64$ when periodic boundary conditions begin to affect results. This could be seen as a shortcoming; perhaps further interesting dynamics would have been found at later spin angles. However, bearing in mind the counterspinning nature of the drivers, we can consider the true rotation of a single source to be $\theta=2 \times 2.64$, already a significant angle compared with observed solar-like flux rotations (see, for example, Brown et al. 2001).

\section{Conclusions}

We have described a simple numerical experiment in which the magnetic footpoints of two, initially potential, intertwined flux tubes are spun, while their positions remain fixed. Magnetic flux is divided into four domains and the footpoint motions act to twist the flux and create current sheets at the boundaries between the domains. A central twisted separator current sheet forms early on in the experiment and a stagnation flow develops. The flow brings oppositely directed flux in toward the separator current sheet and reconnection takes place everywhere along it. The separator current sheet grows in cross-sectional length before its endpoints bifurcate to form Y-type points. In planes of constant height the situation strongly resembles the 2D nonlinear reconnection models of Priest \& Lee (1990), with their fast reconnection rates. Strong jets of plasma flow across the magnetic separatrices and regions of reversed current are found close to the ends of the diffusion region. The full three-dimensionality of the experiment modifies the regime, with the magnetic field having a locally 2D O-type structure and the third, vertical field component being the important one for reconnection. In addition, the field topology is found to be highly complex. One timedependent effect is that the current sheets which initially form along the boundaries between flux domains (i.e. separatrix current sheets) move away from these boundaries as the sources are spun and reconnection begins to occur. They are, at later spin angles, instead identified as contact discontinuities.

In comparing the experiment described here with the non-generic case of aligned magnetic flux tubes described by De Moortel \& Galsgaard (2006b). Any degree of misalignment of the magnetic flux tubes has a significant effect on the magnetic topology of the system, since four flux domains will initially be present (instead of just two in the aligned case). In both experiments a central separator current sheet forms in the centre of the domain and in the misaligned case, the current structure is modified by the presence of four wings of current that initially outline the additional separatrices of the field. Once reconnection begins however, the rate at which magnetic flux changes its connectivity is very similar for both cases. Indeed reconnection in the misaligned case is found to occur only along the central separator current sheet; the extra wings of current are not found to modify the process. These two observations suggest the same reconnection mechanism to be operating in both cases. An important difference is found regarding the onset time for reconnection. It is found that strong currents develop at earlier spin-angles in the misaligned case and that, as a result, magnetic reconnection begins sooner; mapping the relevant spin-angles to coronal timescales the onset time is found to be $0.23 \mathrm{~h}$ in the misaligned case but $0.85 \mathrm{~h}$ in the aligned case. In this series of papers we have considered the two most extreme situations of flux-tube alignment and are able therefore to deduce the implications for any general case. We expect reconnection to begin sooner the more tangled the initial magnetic flux-tubes but for it to proceed at the same rate once initiated.

Acknowledgements. The authors would like to thank Klaus Galsgaard for his numerical code and both him and the members of the St Andrews "reconnection club" for helpful discussions. A.W.S. would like to thank PPARC for a Ph.D. studentship. I.D.M. acknowledges support of a Royal Society University Research Fellowship.

\section{References}

Biskamp, D. 1986, Phys. Fluids, 29, 1520

Brown, D. S., Parnell, C. E., De Luca, E. E., Golub, L., \& Mc Mullen, R. A. 2001, Sol. Phys., 201, 305

Close, R. M., Parnell, C. E., Longcope, D. W., \& Priest, E. R. 2004, ApJ, 612, L81

De Moortel, I., \& Galsgaard, K. 2006a, A\&A, 451, 1101

De Moortel, I., \& Galsgaard, K. 2006b, A\&A, 459, 627

Galsgaard, K., \& Nordlund, A. 1996, JGR, 101, 13445

Galsgaard, K., \& Parnell, C. E. 2005, A\&A, 439, 335

Galsgaard, K., Parnell, C. E., \& Blaziot, J. 2000, A\&A, 362, 395

Green, R. M. 1965, in Solar and Stellar Magnetic Fields, ed. R. Lust (Amsterdam: North Holland), IAU Symp., 22, 398

Haynes, A. L., Parnell, C. E., Galsgaard, K., \& Priest, E. R. 2007, Proc. R. Soc. A, 463, 1097

Hornig, G., \& Priest, E. R. 2003, Phys. Plasmas, 10, 2712

Longcope, D. W. 1996, Sol. Phys., 169, 91

Longcope, D. W. 2001, Phys. Plasmas, 8, 5277

Longcope, D. W., \& Cowley, S. C. 1996, Phys. Plasmas, 3, 2885

Madjarska, M. S., Doyle, J. G., \& van Driel-Gesztelyi, L. 2004, ApJ, 603, L57

Nordlund, A., \& Galsgaard, K. 1997, A 3D MHD code for Parallel Computers, Technical report, Astronomical Observatory, Copenhagen University

Parker, E. N. 1957, J. Geophys. Res., 62, 509

Parker, E. N. 1972, ApJ, 174, 499

Parnell, C. E., \& Galsgaard, K. 2004, A\&A, 428, 595

Pontin, D. I., Hornig, G., \& Priest, E. R. 2004, GAFD, 89, 407

Pontin, D. I., Hornig, G., \& Priest, E. R. 2005, GAFD, 99, 77

Priest, E. R. 1982, Solar Magnetohydrodynamics (Dordrecht: Reidel), 203

Priest, E. R., \& Forbes, T. G. 1986, JGR, 91, 5579

Priest, E. R., \& Lee, L. C. 1990, J. Plasma Phys., 44, 337

Priest, E. R., \& Titov, V. S. 1996, Phis. Trans. R. Soc., 354, 2951

Priest, E. R., Heyvaerts, J. F., \& Title, A. M. 2002, ApJ, 576, 533

Priest, E. R., Longcope, D. W., \& Heyvaerts, J. 2005, ApJ, 624, 1057

Sweet, P. A. 1958, Proc. IAU Symp. 6, ed. Bo Lehnert, CUP, 123

Tsuneta, S. 1996, ApJ, 456, L63

van Ballegooijen, A. A. 1986, ApJ, 311, 1001 